\title{
Atuação do enfermeiro na preservação de vestígios na violência sexual contra a mulher: revisão integrativa
}

\author{
Nurses' performance of trace preservation in sexual violence against women: an integrative review \\ Práctica del enfermero en la preservación de vestigios de la violencia sexual contra la mujer: revisión \\ integrativa
}

Camila Lima Ribeiro ${ }^{1}$ (C)

Ivana Cristina Vieira de Lima Maia² (1) Joice Fabrício de Souza ${ }^{1}$ (D)

Vanessa da Frota Santos ${ }^{3}$ (D) Juliana Sampaio dos Santos ${ }^{3}$ (1) Luiza Jane Eyre de Souza Vieira ${ }^{1}$ (D)

1. Universidade de Fortaleza. Fortaleza, CE, Brasil.

2. Universidade Federal do Ceará. Fortaleza, $\mathrm{CE}$, Brasil.

3. Maternidade-Escola Assis Chateaubriand. Fortaleza, CE, Brasil.

Autor correspondente:

Camila Lima Ribeiro.

E-mail: camila_lima_ribeiro@hotmail.com

Recebido em 03/05/2021.

Aprovado em 11/08/2021.

DOI:https://doi.org/10.1590/2177-9465-EAN-2021-0133

\section{RESUMO}

Objetivo: Analisar os desafios da atuação do enfermeiro na preservação de vestígios nos casos de violência sexual contra a mulher, evidenciados na literatura. Método: Trata-se de uma revisão integrativa, realizada nas bases de dados SCOPUS, Cochrane, LILACS, MEDLINE, CINAHL e no Google Acadêmico, em janeiro de 2021. Utilizaram-se os descritores Sex offenses e Forensic Nursing, combinados com o operador booleano AND. Foram encontrados 207 artigos e selecionados 15. Resultados: Os desafios identificados foram: carência de recursos humanos e de formação em enfermagem forense; formação de enfermeiros para coleta de vestígios; execução limitada de procedimentos para a preservação de vestígios; conflitos entre o papel do enfermeiro no cuidado e na coleta de vestígios; ausência de protocolos ou padronização dos já existentes; subnotificação dos casos de agressão; falta de tempo e medo da responsabilidade legal ou represália; ausência de instrumentos legais que regulamentem as atribuições do enfermeiro e seu papel na cadeia de custódia das provas. Conclusão e implicações para a prática: Ações que visem capacitar os enfermeiros. A criação de protocolos, de forma a ampliar e implementar políticas públicas já existentes, são imprescindíveis para o fortalecimento da atuação do enfermeiro na preservação de vestígios nos casos de violência sexual contra a mulher.

Palavras-chave: Delitos sexuais; Enfermagem forense; Mulheres; Violência contra a Mulher; Revisão.

\begin{abstract}
Objective: to analyze the challenges for nurses' performance of trace preservation in cases of sexual violence against women discussed in the literature. Method: this is an integrative review carried out in the SCOPUS, Cochrane, LILACS, MEDLINE and CINAHL databases and in Google Scholar in January 2021. The descriptors Sex Offenses and Forensic Nursing were used, combined with the Boolean operator AND. A total of 207 articles was found and 15 were selected. Results: The identified challenges were: lack of human resources and training in forensic nursing; training of nurses to collect traces; limited performance of trace preservation procedures; conflicts between the nurse's role in care provision and in the collection of traces; absence of protocols or standardization of the existing ones; underreporting of sexual assault cases; lack of time and fear of liability or reprisal; absence of legal instruments to regulate the nurses' attributions and role in the chain of evidence custody. Conclusion and implications for practice: Actions aimed at training nurses and creating protocols, with expansion and implementation of the existing public policies, are essential to strengthen the role of nurses in trace preservation in cases of sexual violence against women.
\end{abstract}

Keywords: Sex offenses; Forensic nursing; Women; Violence Against Women; Review.

\section{RESUMEN}

Objetivo: analizar los desafíos de la práctica del enfermero en la preservación de vestigios en casos de violencia sexual contra la mujer evidenciados en la literatura. Método: revisión integrativa, realizada en las bases de datos SCOPUS, Cochrane, LILACS, MEDLINE, CINAHL y Google Académico en enero/2021. Se utilizaron los descriptores Sex offenses y Forensic Nursing, combinados con el operador booleano AND. Encontrados 207 artículos; 15 seleccionados. Resultados: Desafíos identificados: falta de recursos humanos y capacitación en enfermería forense; formación de enfermeros para la recogida de rastros; ejecución limitada de procedimientos de conservación de vestigios; conflictos entre el papel del enfermero en el cuidado y en la recolección de huellas; ausencia de protocolos o estandarización de los existentes; subregistro de casos de agresión; falta de tiempo y temor de responsabilidad legal o represalias; ausencia de instrumentos legales que regulen las atribuciones de los enfermeros y su papel en la cadena de custodia probatoria. Conclusión e implicaciones para la práctica: Acciones dirigidas a la formación de enfermeros y elaboración de protocolos con ampliación e implementación de las políticas públicas existentes son fundamentales para fortalecer el papel de los enfermeros en la preservación de vestigios en casos de violencia sexual contra la mujer.

Palabras clave: Delitos sexuales; Enfermería forense; Mujeres; Violencia contra la Mujer; Revisión 


\section{INTRODUÇÃO}

A International Association of Forensic Nursing (IAFN) reconhece a enfermagem forense como uma especialidade que utiliza a ciência da enfermagem em prol da população e da justiça. Seu principal objetivo é oferecer suporte às investigações relativas a vítimas dos vários tipos de violências - a exemplo da violência física, sexual, psicológica, econômica e doméstica -, além de acidentes traumáticos, na ocorrência de óbitos e/ou no tratamento do trauma de agressores e vítimas, tráfico humano, dentre outras situações ${ }^{1,2}$.

Reconhecida no cenário internacional, a especialidade forense abrange diversas áreas de competência, alcançando importância ímpar na atenção à saúde integral das vítimas de violência, pessoas e sobreviventes de desastres de massa, pessoas em privação de liberdade inseridas no sistema prisional, nos serviços de saúde mental e nos setores afins. No Brasil, apesar de a especialidade ser reconhecida pelo Conselho Federal de Enfermagem desde 2011, há necessidade de divulgar e concretizar a profissão no país, visto que representa uma demanda da sociedade atual ${ }^{3}$.

A violência é reconhecida como um grave problema social, de proporções crescentes, e desafia os órgãos internacionais na busca de soluções para mitigar as ocorrências e seus desdobramentos. Nesse sentido, países como Estados Unidos e Canadá passaram a elaborar e promulgar políticas governamentais e a aprimorar a aplicação da ciência forense, qualificando e aperfeiçoando a coleta de vestígios, visando preservar a vida de pessoas e responsabilizar os autores desses casos ${ }^{4}$.

Reportando-se aos casos de delitos sexuais, o enfermeiro forense ancora-se no conhecimento técnico-científico e nas habilidades que perpassam uma formação humanística. Assim, poderá contribuir, sobremaneira, com o Poder Judiciário - tanto nas investigações quanto no enfrentamento a essa modalidade de crime -, evidenciando sua relevância para a ciência forense, estabelecendo importante elo entre o setor de Saúde e o Judiciário $^{5}$. Ressalta-se ainda que, de acordo com a Portaria $n$. $^{\circ}$ 204, de 2016, do Ministério da Saúde, a Resolução n. ํ 564, de 2017, do Conselho Federal de Enfermagem (COFEN), dispõe sobre o dever da enfermagem ao atuar no atendimento de casos de violência contra mulher, afirmando, no art. 52, que quaisquer episódios envolvendo violências precisam ser notificados ${ }^{6-9}$.

No decorrer dos atendimentos, é consenso que os vestígios são vitais para o sucesso de uma investigação, pois contribuem para a descoberta de uma solução que conduza à resolução dos processos-crime. A investigação criminal procura chegar à verdade, com vista à perseguição penal dos agentes do crime. Assim, a análise dos vestígios é decisiva para provar a culpa ou a inocência de determinado suspeito ${ }^{10}$.

As situações que envolvem crime violento e traumas geram consequências nas quais se faz necessária a atuação interdisciplinar. Os enfermeiros têm como principal objetivo a assistência à vítima de violência contra a mulher, promovendo cuidados de saúde. Dessa forma, é imprescindível que esses profissionais realizem a identificação apropriada, com adequada preservação dos vestígios (biológicos e não biológicos) encontrados no corpo da vítima/agressor ou no local da ocorrência do crime, que futuramente poderão ser alvo de prova pericial em Tribunal com relevância Médico-Legal ${ }^{11}$.

Nesse contexto, torna-se imperiosa a incorporação da prática clínica do enfermeiro forense no cotidiano dos profissionais que atendem pessoas expostas às violências, cenário cada vez mais presente nos serviços de saúde. Este estudo baseou-se na seguinte questão: "Quais são as evidências acerca dos desafios da atuação do enfermeiro na preservação de vestígios nos casos de violência sexual contra a mulher?". Ponderando que manuseios inadequados e/ou a supressão de vestígios inviabilizam o processo investigativo, estudos com esse enfoque são fundamentais para aprofundar o conhecimento sobre a temática. $O$ propósito é explicitar a importância da atuação do enfermeiro nesses cenários e contibuir para viabilizar a preservação de vestígios em caso de violência sexual a fim de assegurar lisura ao fluxo de investigação do crime e reponsabilização dos perpetradores.

Nesse constructo, o estudo tem como objetivo analisar os desafios da atuação do enfermeiro na preservação de vestígios nos casos de violência sexual contra a mulher, evidenciados na literatura.

\section{MÉTODO}

Trata-se de uma revisão integrativa orientada pelas etapas propostas por Mendes et al. ${ }^{12}: 1$. $^{\text {a }}$ etapa: identificação do tema e seleção da hipótese ou questão de pesquisa para a elaboração da revisão integrativa; 2. a etapa: estabelecimento de critérios para inclusão e exclusão de estudos/amostragem ou busca na literatura; 3. ${ }^{a}$ etapa: definição das informações a serem extraídas dos estudos selecionados/categorização dos estudos; 4. ${ }^{\text {a }}$ etapa: avaliação dos estudos incluídos na revisão integrativa; $5 .{ }^{\text {a }}$ etapa: interpretação dos resultados; 6. ${ }^{\text {a }}$ etapa: apresentação da revisão/ síntese do conhecimento.

A questão norteadora da presente revisão foi: "Quais são as evidências acerca dos desafios da atuação do enfermeiro na preservação de vestígios nos casos de violência sexual contra a mulher?". Esta questão foi definida conforme o acrônimo P.I.Co ( $\mathrm{P}$ = Participante, $\mathrm{I}=$ Fenômeno de interesse, $\mathrm{Co}=$ Contexto do estudo $)^{13}$. Neste estudo, o participante é representado pelo enfermeiro generalista e/ou forense; o fenômeno de interesse são as mulheres em situação de violência sexual; o contexto refere-se ao nível pré-hospitalar e hospitalar.

A análise das evidências da literatura científica sobre a temática foi realizada no mês de janeiro de 2021, nas seguintes bases de dados, acessadas por meio do Portal da Coordenação de Aperfeiçoamento de Pessoal de Nível Superior (CAPES): SciVerse Scopus (SCOPUS), Cochrane Library (Cochrane), Latin American and Caribbean Literature in Health Sciences (LILACS), MEDLINE via PubMed, Cumulative Index to Nursing and Allied Health Literature (CINAHL). Também se realizou a busca no Google Acadêmico (Google Scholar). Utilizaram-se os descritores "Sex offenses" e "Forensic Nursing" cadastrados nos DeCS (Descritores em Ciências da Saúde) e no MeSH (Medical Subject Heading), combinados com o operador booleano AND.

Os critérios de inclusão utilizados foram: artigos originais, textos como opinião de especialista, de atualização ou teórico, 
disponíveis na íntegra, publicados no idioma português, inglês ou espanhol, entre os anos de 2011 e 2021, e que abordassem a temática desta pesquisa. Os critérios de exclusão estabelecidos foram: artigos sobre outros tipos de violência, reportagens, estudos de desenvolvimento de tecnologias.

A seleção dos artigos ocorreu em dois momentos. Inicialmente, foi realizada a leitura minuciosa dos títulos e dos resumos dos 207 estudos encontrados nas bases de dados supracitadas. Após aplicar os critérios de inclusão e efetuar a exclusão de 5 artigos duplicados, restaram 29 artigos. Posteriormente, realizou-se a leitura na íntegra dos textos, de modo exaustivo, com o intuito de analisar se atendiam aos requisitos e critérios de inclusão relacionados à temática abordada na revisão integrativa. Nessas duas etapas, ocorreu participação de dois pesquisadores que atuaram de forma independente. Em caso de desacordo entre os pesquisadores, houve diálogo para que entrassem em um consenso. A amostra final foi composta por 15 estudos (Figura 1).

A análise dos dados extraídos nos estudos selecionados foi realizada de forma descritiva, resultando na criação de um quadro sinóptico de caracterização dos estudos (autores, ano, país de realização do estudo, participantes, métodos, principais resultados e nível de evidência). Para classificação do nível de evidência, utilizou-se a hierarquia de evidências proposta por Melnyk e Fineout-Overholt ${ }^{14}$ : revisões sistemáticas e metanálise de ensaios clínicos randomizados, nível I; ensaios clínicos randomizados, nível II ; ensaio controlado não randomizado, nível III; estudos caso-controle ou coorte, nível IV; revisões sistemáticas de estudos qualitativos e descritivos, nívelV; estudos qualitativos ou descritivos, nível Vl; e parecer de autoridades e/ou relatórios de comitês de especialistas, nível VII. Essa hierarquia classifica os níveis I e II como fortes, III a V, moderados, e VI a VII, fracos.

Ressalta-se que os resultados obtidos na revisão integrativa foram discutidos com base na literatura científica sobre o tema, respeitando a integridade das informações apresentadas nos artigos e os direitos autorais, havendo o devido cuidado para não realizar mudanças no conteúdo encontrado em benefício desta pesquisa.

\section{RESULTADOS}

Os 15 estudos selecionados foram desenvolvidos nos seguintes países: Brasil, Estados Unidos, Índia e Inglaterra. Houve maior representatividade de estudos publicados nos Estados Unidos $(n=8)$ e no Brasil $(n=5)$. Em relação ao número de publicação, variou de 2011 a 2019, com maior número de artigos publicados em $2014(n=5)$. Quanto ao tipo de estudo, todos foram qualitativos ou descritivos ( $n=15)$, e 14 tinham nível de evidência VI. Referente à abordagem metodológica, encontraram-se: estudos qualitativos (8), estudos quantitativos (4) e estudos métodos mistos (3) (Quadro 1).

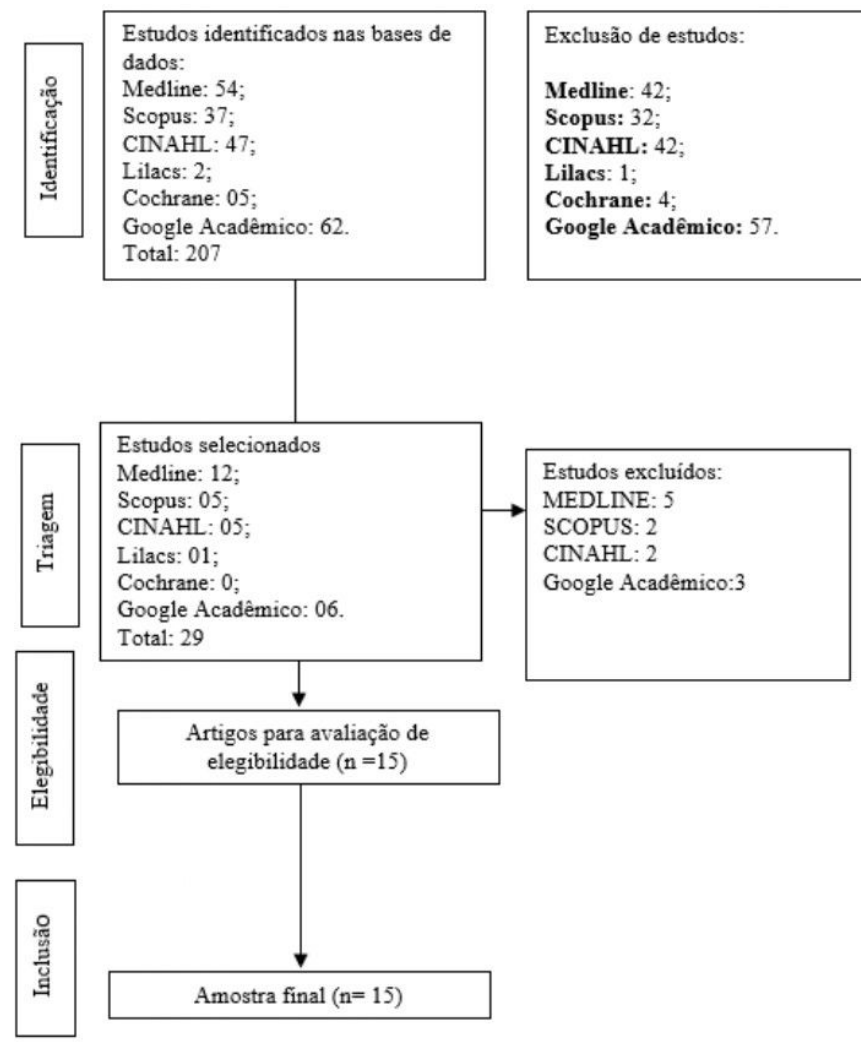

Figura 1. Fluxograma de descrição dos artigos encontrados, excluídos e selecionados de acordo com cada base de dados. Elaborada pelas autoras. Fortaleza-Ceará, 2021.

Fonte: Elaborada pelas autoras 


\section{Preservação de vestígios na violência sexual}

Ribeiro CL, Maia ICVL, Souza JF, Santos VF, Santos JS, Vieira JLES

Quadro 1. Caracterização dos estudos quanto ao título, autores, ano, país, objetivo, tipo de estudo, principais resultados e nível de evidência

\begin{tabular}{|c|c|c|c|c|c|c|c|}
\hline $\mathrm{N}$ & Título & Autores/ ano & País & $\begin{array}{l}\text { Público-alvo/ } \\
\text { amostra }\end{array}$ & Objetivo & Tipo de estudo & $\begin{array}{l}\text { Nível de } \\
\text { evidência }\end{array}$ \\
\hline 1 & $\begin{array}{l}\text { O enfermeiro e a preservação } \\
\text { de vestígios frente à violência } \\
\text { sexual contra a mulher }\end{array}$ & $\begin{array}{l}\text { Souza et al. } \\
\qquad(2017)^{15}\end{array}$ & Brasil & $\begin{array}{c}15 \text { enfermeiros } \\
\text { generalistas }\end{array}$ & $\begin{array}{l}\text { Investigar a preservação de } \\
\text { vestígios pelo enfermeiro durante } \\
\text { o atendimento de mulheres vítimas } \\
\text { de violência sexual em um serviço } \\
\text { de urgência e emergência do estado } \\
\text { de Sergipe. }\end{array}$ & $\begin{array}{l}\text { Analítico e } \\
\text { descritivo, } \\
\text { quantitativo }\end{array}$ & Nível VI \\
\hline 2 & $\begin{array}{c}\text { Preservation of Forensic } \\
\text { Evidence by Nurses in a } \\
\text { Prehospital Emergency Care } \\
\text { Service in Brazil }\end{array}$ & $\begin{array}{l}\text { Silva et al. } \\
(2020)^{16}\end{array}$ & Brasil & $\begin{array}{c}128 \\
\text { enfermeiros } \\
\text { generalistas }\end{array}$ & $\begin{array}{c}\text { Avaliar a relação entre o } \\
\text { conhecimento dos enfermeiros e o } \\
\text { desempenho dos procedimentos de } \\
\text { evidências forenses. }\end{array}$ & $\begin{array}{c}\text { Estudo } \\
\text { transversal } \\
\text { quantitativo }\end{array}$ & Nível VI \\
\hline 3 & $\begin{array}{l}\text { Defining the boundaries: How } \\
\text { sexual assault nurse examiners } \\
\text { (SANEs) balance patient } \\
\text { care and law enforcement } \\
\text { collaboration }\end{array}$ & $\begin{array}{l}\text { Campbell et al. } \\
\qquad(2011)^{17}\end{array}$ & Estados Unidos & $\begin{array}{l}11 \text { enfermeiros } \\
\text { forenses }\end{array}$ & $\begin{array}{l}\text { Compreender como os enfermeiros } \\
\text { que atuam na realização de exame } \\
\text { forense, nos casos de agressão } \\
\text { sexual, definem seu trabalho com } \\
\text { seus pacientes, como colaboram } \\
\text { com a aplicação da lei e como } \\
\text { negociam a diferenciação de papéis. }\end{array}$ & $\begin{array}{l}\text { Qualitativo e } \\
\text { quantitativo }\end{array}$ & Nível V \\
\hline 4 & $\begin{array}{l}\text { Forensic nursing e Global } \\
\text { scenario and Indian perspective }\end{array}$ & $\begin{array}{l}\text { Dash et al. } \\
(2016)^{18}\end{array}$ & Índia & $\begin{array}{l}\text { Amostra não } \\
\text { informada, } \\
\text { enfermeiros } \\
\text { forenses }\end{array}$ & $\begin{array}{l}\text { Analisar o trabalho da Enfermagem } \\
\text { Forense perante vítimas de violência } \\
\text { sexual no cenário global e sua } \\
\text { perspectiva na Índia. }\end{array}$ & Qualitativo & Nível VI \\
\hline 5 & $\begin{array}{l}\text { Original research: Giving sexual } \\
\text { assault survivors time to decide: } \\
\text { an exploration of the use and } \\
\text { effects of the nonreport option }\end{array}$ & $\begin{array}{l}\text { Heffron et al. } \\
\qquad(2014)^{19}\end{array}$ & Estados Unidos & $\begin{array}{l}79 \text { enfermeiros } \\
\text { forenses }\end{array}$ & $\begin{array}{l}\text { Examinar a implementação da opção } \\
\text { de não notificação da violência sexual } \\
\text { no Texas; explorar seu impacto sobre } \\
\text { enfermeiras que atuam na realização } \\
\text { de exame forense, nos casos de } \\
\text { agressão sexual e o sistema de justiça } \\
\text { criminal; e identificar os pontos } \\
\text { fortes e os desafios do processo de } \\
\text { não notificação. }\end{array}$ & $\begin{array}{l}\text { Quantitativo e } \\
\text { qualitativo }\end{array}$ & Nível V \\
\hline 6 & $\begin{array}{l}\text { Sexual Assault Nurse Examiner } \\
\text { Forensic Examinations for } \\
\text { Immigrant Victims: A Case Study }\end{array}$ & Payne $(2018)^{20}$ & Estados Unidos & $\begin{array}{l}\text { Amostra não } \\
\text { informada, } \\
\text { enfermeiros } \\
\text { forenses }\end{array}$ & $\begin{array}{l}\text { Analisar a atuação dos enfermeiros } \\
\text { forenses que atuam na realização } \\
\text { de exame forense, nos casos de } \\
\text { agressão sexual, no atendimento às } \\
\text { vítimas de imigrantes nos Estados } \\
\text { Unidos. }\end{array}$ & Estudo de caso & Nível VI \\
\hline 7 & $\begin{array}{l}\text { The Role of the Sexual Assault } \\
\text { Nurse Examiner in England: } \\
\text { Nurse Experiences and } \\
\text { Perspectives }\end{array}$ & $\begin{array}{l}\text { Cowley et al. } \\
\qquad(2014)^{21}\end{array}$ & Inglaterra & $\begin{array}{l}5 \text { enfermeiros } \\
\text { forenses }\end{array}$ & $\begin{array}{l}\text { Analisar o papel dos enfermeiros } \\
\text { forenses que atuam na realização } \\
\text { de exame forense, nos casos de } \\
\text { agressão sexual na Inglaterra. }\end{array}$ & Qualitativo & Nível VI \\
\hline 8 & $\begin{array}{l}\text { Client Satisfaction With Nursing- } \\
\text { led Sexual Assault and Domestic } \\
\text { Violence Services in Ontario }\end{array}$ & $\begin{array}{l}\text { Du Mont et al. } \\
\qquad(2014)^{22}\end{array}$ & Estados Unidos & $\begin{array}{l}35 \text { enfermeiros } \\
\text { forenses }\end{array}$ & $\begin{array}{l}\text { Avaliar a satisfação dos pacientes } \\
\text { atendidos pelos serviços de agressão } \\
\text { e violência doméstica, liderados por } \\
\text { enfermagem, em Ontário }\end{array}$ & $\begin{array}{c}\text { Quantitativo e } \\
\text { qualitativo }\end{array}$ & Nível V \\
\hline 9 & $\begin{array}{c}\text { The perception of role conflict } \\
\text { in sexual assault nursing and its } \\
\text { effects on care delivery }\end{array}$ & $\begin{array}{c}\text { Downing e } \\
\text { Mackin }(2012)^{23}\end{array}$ & Estados Unidos & $\begin{array}{l}14 \text { enfermeiros } \\
\text { forenses }\end{array}$ & $\begin{array}{l}\text { Compreender a percepção do } \\
\text { conflito de papéis na enfermagem } \\
\text { de agressão sexual e seu impacto } \\
\text { na atuação dos enfermeiros e na } \\
\text { prestação de cuidados. }\end{array}$ & Qualitativo & Nível VI \\
\hline
\end{tabular}

Fonte: Elaborado pelas autoras 
Quadro 1. Continuação...

\begin{tabular}{|c|c|c|c|c|c|c|c|}
\hline $\mathrm{N}$ & Título & Autores/ano & País & $\begin{array}{l}\text { Público-alvo/ } \\
\text { amostra }\end{array}$ & Objetivo & Tipo de estudo & $\begin{array}{l}\text { Nível de } \\
\text { evidência }\end{array}$ \\
\hline 10 & $\begin{array}{l}\text { When There is No Sexual Assault } \\
\text { Nurse Examiner: Emergency } \\
\text { Nursing Care for Female Adult } \\
\text { Sexual Assault Patients }\end{array}$ & $\begin{array}{c}\text { Delgadillo } \\
(2017)^{24}\end{array}$ & Estados Unidos & Não se aplica & $\begin{array}{l}\text { Fornecer, aos enfermeiros do } \\
\text { pronto-socorro que não são } \\
\text { especialistas na área forense, } \\
\text { uma visão geral das habilidades } \\
\text { necessárias para cuidar e tratar } \\
\text { pacientes vítimas de violência no } \\
\text { serviço de emergência. }\end{array}$ & Texto teórico & Não se aplica \\
\hline 11 & $\begin{array}{l}\text { The Medical/Legal Aspects of } \\
\text { Sexual Assault Nurse Examiner } \\
\text { (SANE) Programs in Emergency } \\
\text { Departments }\end{array}$ & Bimber $(2014)^{25}$ & Estados Unidos & Não se aplica & $\begin{array}{l}\text { Compreender a importância das } \\
\text { enfemeiras forenses no serviço de } \\
\text { emergência. }\end{array}$ & Texto teórico & Não se aplica \\
\hline 12 & Forensic Nursing: Focus on SANE & $\begin{array}{c}\text { Cataruozolo } \\
(2015)^{26}\end{array}$ & Estados Unidos & Não se aplica & $\begin{array}{l}\text { Descrever, resumidamente, a } \\
\text { especialidade de enfermagem } \\
\text { forense, em especial os enfermeiros } \\
\text { que atuam na realização de exame } \\
\text { forense nos casos de agressão } \\
\text { sexual. }\end{array}$ & Texto teórico & Não se aplica \\
\hline 13 & $\begin{array}{l}\text { Conhecimento e atuação } \\
\text { de profissionais de saúde } \\
\text { na preservação de vestígios } \\
\text { forenses em pacientes vítimas } \\
\text { de violência sexual, em hospital } \\
\text { de trauma em Aracaju }\end{array}$ & $\begin{array}{c}\text { Santos et al. } \\
(2019)^{27}\end{array}$ & Brasil & $\begin{array}{c}33 \text { enfermeiros } \\
\text { generalistas }\end{array}$ & $\begin{array}{l}\text { Analisar o grau de conhecimento } \\
\text { e executabiliade dos profissionais } \\
\text { de saúde na preservação dos } \\
\text { vestígios forenses, no atendimento a } \\
\text { pacientes vítimas de violência sexual } \\
\text { no hospital de trauma de Aracaju }\end{array}$ & $\begin{array}{c}\text { Analítico,descritivo } \\
\text { e quantitativo }\end{array}$ & Nível VI \\
\hline 14 & $\begin{array}{l}\text { Preservação de vestígios no pré- } \\
\text { hospitalar: papel das equipes de } \\
\text { emergência médica em cenários } \\
\text { forenses }\end{array}$ & Gomes $(2018)^{28}$ & Brasil & Não informado & $\begin{array}{l}\text { Apresentar as diversas situações } \\
\text { criminógenas que ocorrem no } \\
\text { contexto pré-hospitalar e apresentar } \\
\text { os respectivos vestígios que devem } \\
\text { ser preservados. }\end{array}$ & Qualitativo & Nível VI \\
\hline 15 & $\begin{array}{l}\text { Preservation of forensic traces } \\
\text { by health professionals in a } \\
\text { hospital in Northeast Brazil }\end{array}$ & $\begin{array}{l}\text { De Oliveira } \\
\text { Musse et al. } \\
(2020)^{29}\end{array}$ & Brasil & $\begin{array}{c}33 \text { enfermeiros } \\
\text { generalistas }\end{array}$ & $\begin{array}{l}\text { Descrever o conhecimento dos } \\
\text { profissionais que atuam no pronto- } \\
\text { socorro do Hospital de Urgência } \\
\text { de Sergipe (HUSE), no estado de } \\
\text { Sergipe, Nordeste do Brasil, sobre a } \\
\text { preservação de vestígios de perícia } \\
\text { e sua capacidade de implementar } \\
\text { na prática os processos relacionados } \\
\text { necessários. }\end{array}$ & Quantitativo & Nível VI \\
\hline
\end{tabular}

Fonte: Elaborado pelas autoras

O Quadro 2 apresenta as evidências da literatura científica sobre os desafios da atuação do enfermeiro na preservação de vestígios, nos casos de violência sexual contra a mulher.

\section{DISCUSSÃO}

A violência tornou-se um problema de saúde pública global. Os serviços de emergência dos hospitais frequentemente recebem vítimas de diferentes tipos de violência. Dessa forma, os profissionais de saúde, em especial os enfermeiros, que, por habitualmente prestarem o primeiro contato com as vítimas, desempenham função indispensável, contribuindo nas investigações e auxiliando a justiça. Em 2013, recomendações e protocolos para profissionais de saúde foram criados com intuito de contemplar vítimas de violência sexual, e para outros tipos de violência, no ano de $2015^{27}$.

A presente revisão apontou, dentre os desafios do papel do enfermeiro, nesse contexto, a carência de recursos humanos e de formação em enfermagem forense para atendimento de casos de violência sexual contra a mulher. Corroborando esse 
Quadro 2. Evidências na literatura científica sobre os desafios da atuação do enfermeiro na preservação de vestígios em mulheres vítimas de violência sexual

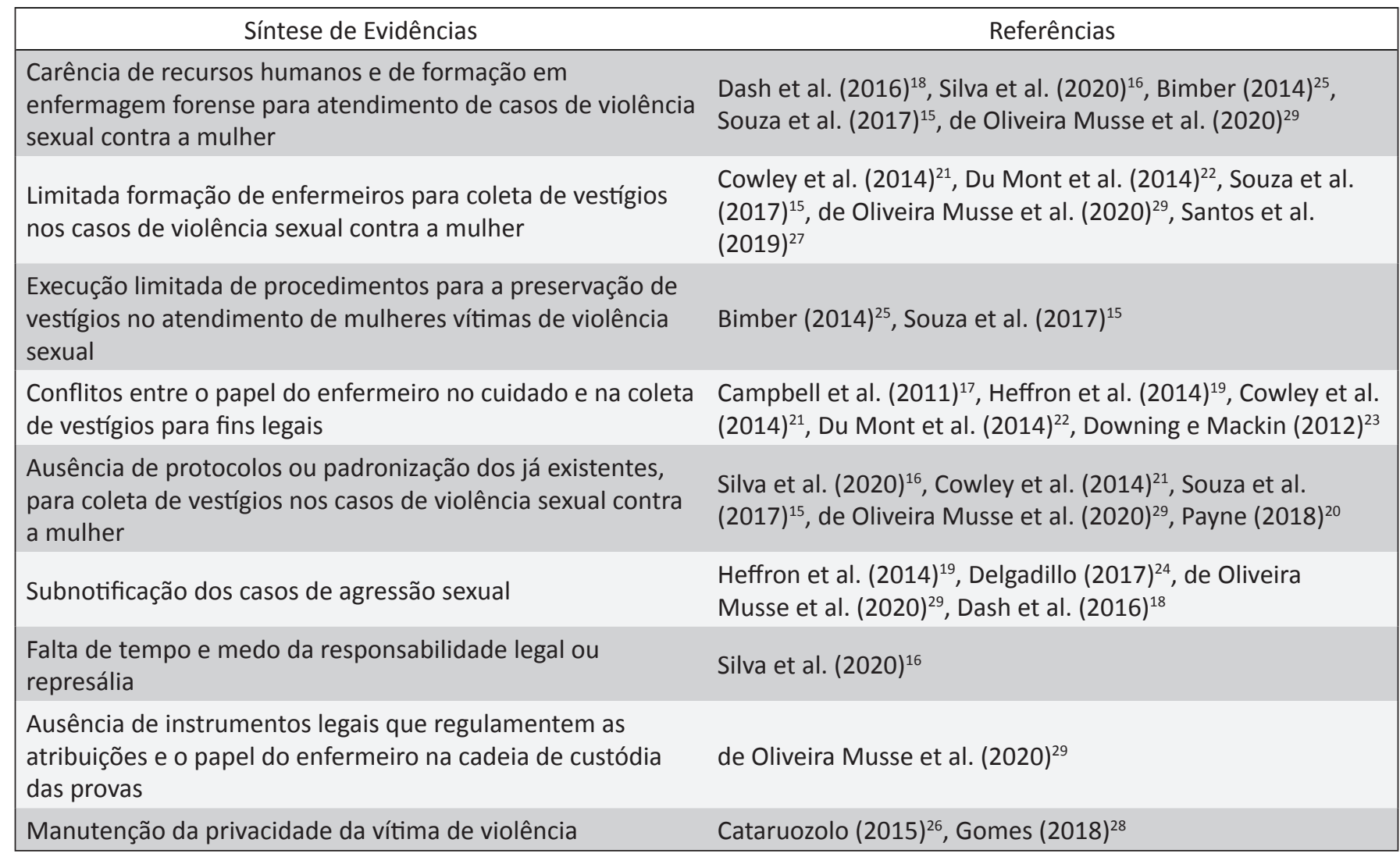

Fonte: Elaborado pelas autoras

resultado, evidência demonstra que o campo de trabalho da enfermagem forense brasileira precisa ser conquistado, pois algumas subespecialidades - como a preservação de vestígios -, ainda é tradicionalmente realizada pela polícia criminal ${ }^{27}$.

Além disso, diferentemente de países como Inglaterra e EUA, a enfermagem forense no Brasil ainda é pouco conhecida e possui escassos centros de especialização ${ }^{20,21}$. De modo geral, encontra-se em desenvolvimento inicial na América Latina; no entanto, é reconhecida como especialização para o profissional enfermeiro(a), pelo Conselho Federal, desde 2011, mediante a Resolução n. ํ 389, de 2011, revogada, posteriormente, pela Resolução n. ${ }^{\circ}$ 0581, de $2018^{15}$.

Nota-se que os enfermeiros forenses possuem um amplo campo de trabalho, podendo atuar em diversas áreas envolvendo violência doméstica e sexual, abuso/negligência infantil, maus-tratos, enfermagem carcerária, investigação de óbitos e desastres em massa. Além das áreas de prática forense que inclue enfermagem psiquiátrica, testemunho pericial, consultoria, saúde pública e segurança, serviços de trauma e emergência, entre outras situações. Utilizam, nesse sentido, habilidades exclusivas para realizar um plano de cuidado holístico, com vistas a responder as necessidades físicas e psicossociais da vítima ${ }^{26,28}$.

Um dos aspectos revelados pela presente revisão foi a ausência de protocolos - ou padronização dos já existentes - para coleta de vestígios nos casos de violência sexual contra a mulher, demonstrando a necessidade de estudos com foco no desenvolvimento desse tipo de tecnologia. Conforme apontado pela literatura, o enfermeiro forense deve realizar a entrevista das vítimas de agressão sexual em local adequado, buscando proporcionar segurança, conforto, confidencialidade, e respeitando sua situação, longe de qualquer interrupção ou intrusão. A avaliação inicial inclui: entrevista; exame físico da cabeça aos pés; identificação da história pessoal e médica; acesso a documentação relevante; realização de testes diagnósticos; observação e colaboração com outros profissionais de saúde; além da identificação, coleta e preservação de vestígios forenses ${ }^{10}$.

A literatura indica que o enfermeiro examinador de agressão sexual (SANE) realiza um atendimento mais abrangente do que outros profissionais de saúde. Ademais, sua atuação gera economia de custos, fortalecimento das leis a favor das vítimas, além de ser um modelo de atendimento bem-sucedido e visto com bons olhos pelas autoridades policiais ${ }^{21}$.

No Brasil, embora a notificação dos casos suspeitos e confirmados de violência seja uma ação compulsória, ainda se destaca, como desafio, a subnotificação dos casos de agressão sexual, uma questão evidenciada na presente revisão ${ }^{18,19,24,29}$. Tal fato pode estar relacionado ao medo da responsabilidade legal ou represália por parte do profissional ${ }^{16}$. Assim, a assistência 
de enfermagem, nesses casos, restringe-se ao cuidado com as lesões, contribuindo com o subdiagnóstico e o subdimensionamento dos casos de violência.

Nossos achados revelaram resultados positivos da assistência quando realizada por enfermeiro forense examinador de agressão sexual, como: redução no tempo de espera do atendimento; registro, coleta e preservação adequada dos vestígios forenses; $\mathrm{e}$ uma maior confidencialidade da vítimas. Protocolos institucionais orientam o atendimento as vítimas e confirmam o valor do cuidado prestado por meio dos feedbacks, maior aceitação de coleta de vestígios e denúncia dos casos a justiça ${ }^{20,22}$.

Programas de atendimento a vítimas de agressão sexual realizados por enfermeiros forenses - habilitados ou treinados oferecem alta qualidade e atendem às necessidades de saúde mental e física dos pacientes, abrangendo coleta de evidências, profilaxia de gravidez e infecções sexualmente transmissíveis, que garantem o tratamento adequado, além de promover a prevenção de lesões futuras ${ }^{25}$.

Há evidências de que, embora assumam postura neutra durante $o$ atendimento, pacientes que foram assistidos por enfermeiros forenses têm maior segurança em denunciar os casos às autoridades policiais, pois, além de diminuir sentimentos de culpa e impotência das vítimas, tais profissionais enaltecem seus pontos fortes e prestam-Ihes orientações durante o atendimento, aliviando seus temores ${ }^{17}$.

Os serviços de saúde constantemente recebem pessoas que foram acometidas por diversas situações de violência. Entretanto, na literatura pesquisada, destaca-se a presença de serviços especializados de enfermagem para atender vítimas de agressão sexual ocorrida em vários países; diferente do Brasil, onde há escassez de conhecimento acerca do assunto e despreparo dos profissionais. Por atuar na linha de frente da assistência, os enfermeiros generalistas prestam os primeiros cuidados às vítimas; no entanto, nem sempre estão preparados para lidar com esse cenário ${ }^{15}$. Ressalta-se que, caso os vestígios não sejam coletados e manuseados de maneira adequada, compromete qualquer análise forense e investigação legal ${ }^{29}$.

Quanto à abordagem das competências legais e à realização de capacitação dos profissionais generalistas em nível nacional, ainda encontram-se lacunas que dificultam a realização dos atendimentos. Isso porque, embora haja normativas do Sistema Único de Saúde que regulamentam a coleta de vestígios nas suas unidades, não existe uma portaria que reconheça o profissional da área na cadeia de custódia das provas e validação dos vestígios pelos institutos de criminalística ${ }^{27}$. Esse aspecto foi evidenciado pelos resultados de um estudo nacional que compôs a presente revisão ${ }^{29}$.

Conforme evidenciado nesta pesquisa, um dos desafios vivenciados na prática são os conflitos entre o papel do enfermeiro no cuidado e na coleta de vestígios para fins legais. Devido à falta de política, restrições econômicas ou falta de incentivos aos programas de atendimento a vítimas de agressão sexual, o enfermeiro forense examinador de agressão sexual nem sempre está disponível para cuidar da referida clientela. Dessa forma, o enfermeiro generalista que recebe e presta os primeiros atendimentos necessita possuir conhecimento e ferramentas que facilitam a condução do caso ${ }^{24}$.

Os estudos selecionados demonstraram, como um importante desafio, a limitada formação de enfermeiros para coleta de vestígios nos casos de violência sexual contra a mulher ${ }^{15,21,22,29}$ Embora os enfermeiros generalistas considerem importantes os procedimentos de evidência forense frente ao atendimento à mulher vítima de violência sexual, não se sentem preparados técnica e cientificamente para desempenhar tal função, deixando evidente a necessidade de treinamento e adesão de protocolos institucionais. ${ }^{16}$ Essa limitada formação resulta em outro desafio evidenciado na presente revisão - a execução restrita de procedimentos para a preservação de vestígios no atendimento de mulheres vítimas de violência sexual ${ }^{15,25}$.

Segundo estudo nacional realizado com a equipe multiprofissional em um hospital de trauma, a maioria dos profissionais não se sentia preparada para realizar a preservação e coleta de vestígios, e a maior parte dos procedimentos listados não era executada pelos profissionais, embora fossem conhecidos. Verificou-se também que as ações mais executadas foram aquelas relacionadas à documentação, divergindo dos procedimentos referentes à coleta de vestígios que apresentou menor execução27. Além disso, a dificuldade na manutenção da privacidade da vítima durante o atendimento foi outro desafio citado, fato que pode prejudicar a coleta de informações cruciais do caso $^{26,28}$.

Ressalta-se ainda que a necessidade de treinamento da equipe e a inclusão do assunto nos cursos de graduação é um problema vivenciado não apenas no Brasil. Estudo realizado na Índia relata a mesma carência, traçando um paralelo entre a assistência de enfermagem prestada às vítimas - por enfermeiras capacitadas e com carreiras consolidadas - em países desenvolvidos e a realidade vivenciada nos países subdesenvolvidos e emergentes ${ }^{18}$. Além disso, o estudo apontou que os enfermeiros examinadores de agressão sexual apresentaram maior percentual de desenvolver a Síndrome de Burnout, e menor percentual no conflito de papéis fato associado à insatisfação e esgotamento no trabalho ${ }^{23}$.

\section{CONCLUSÃO}

Constatou-se que, não obstante ser uma necessidade natural dos serviços de emergência, a enfermagem forense ainda é pouco divulgada em nível nacional, sendo muitas vezes os enfermeiros os primeiros a prestarem atendimento às vítimas de violência. Todavia, apesar de compreenderem a importância da cadeia de custódia, não se sentem preparados para atender às vítimas de violência sexual, por isso a capacitação é uma das necessidades mais citadas nos estudos.

Aponta-se para a necessidade da criação de protocolos que direcionem as ações e condutas dos envolvidos no atendimento, bem como a capacitação dos enfermeiros. A mudança na diretriz curricular dos cursos de enfermagem, com inclusão da temática na matriz curricular, levando em consideração a indispensabilidade do conhecimento a ser adquirido ainda na 
formação acadêmica, resulta na excelência de profissionais para atuarem frente a vítimas de violência sexual.

Outrossim, salienta-se a importância da criação de legislações que garantam a realização da cadeia de custódia por enfermeiros forenses, práticas realizadas atualmente, em nosso país, por outros profissionais. Dessa maneira, destaca-se a ampliação e implementação de políticas públicas já existentes, assim como o envolvimento de entidades capacitadas, visando estratégias de fortalecimento da visibilidade da especialidade de enfermeiro forense no âmbito brasileiro.

Esta pesquisa ofereceu limitações no que diz respeito à quantidade reduzida de estudos primários relacionados aos desafios da atuação do enfermeiro na preservação de vestígios nos casos de violência sexual contra a mulher; entretanto, por mais que tenham sido escassos, contribuiram de forma inegável para a construção de um diálogo coeso.

\section{CONTRIBUIÇÕES DOS AUTORES}

Desenho do estudo. Camila Lima Ribeiro. Ivana Cristina Vieira de Lima Maia. Luiza Jane Eyre de Souza Vieira.

Coleta ou produção dos dados. Camila Lima Ribeiro. Ivana Cristina Vieira de Lima Maia. Luiza Jane Eyre de Souza Vieira.

Análise de dados. Camila Lima Ribeiro. Ivana Cristina Vieira de Lima Maia. Joice Fabrício de Souza. Vanessa da Frota Santos. Juliana Sampaio dos Santos. Luiza Jane Eyre de Souza Vieira.

Interpretação dos resultados. Camila Lima Ribeiro. Ivana Cristina Vieira de Lima Maia. Joice Fabrício de Souza. Vanessa da Frota Santos. Juliana Sampaio dos Santos. Luiza Jane Eyre de Souza Vieira.

Redação e revisão crítica do manuscrito. Camila Lima Ribeiro. Ivana Cristina Vieira de Lima Maia. Joice Fabrício de Souza. Vanessa da Frota Santos. Juliana Sampaio dos Santos. Luiza Jane Eyre de Souza Vieira.

Aprovação da versão final do artigo. Camila Lima Ribeiro. Ivana Cristina Vieira de Lima Maia. Joice Fabrício de Souza. Vanessa da Frota Santos. Juliana Sampaio dos Santos. Luiza Jane Eyre de Souza Vieira

Responsabilidade por todos os aspectos do conteúdo e a integridade do artigo publicado. Camila Lima Ribeiro. Ivana Cristina Vieira de Lima Maia. Joice Fabrício de Souza. Vanessa da Frota Santos. Juliana Sampaio dos Santos. Luiza Jane Eyre de Souza Vieira.

\section{EDITOR CIENTÍFICO}

Ivone Evangelista Cabral (D)

\section{EDITOR ASSOCIADO}

\author{
Stela Maris de Mello Padoin (D)
}

\section{REFERÊNCIAS}

1. Early S. North America's Evolving Nursing Specialty. The Journal of Legal Nurse Consulting [Internet]. 2016; [citado 2021 jun 8];27(4):1015. Disponível em: http://www.aalnc.org/d/do/678
2. Camilo LSS, Dantas TO, Musse JO, Silva DP, Assis ES. Preservação da cena de crime pelo enfermeiro no Serviço de Atendimento Móvel de Urgência: uma revisão integrativa. Caderno de Graduação - Ciências Biológicas e da Saúde. 2017 out; [citado 2021 mar 17];4(2):185-201. Disponível em: https://periodicos.set.edu.br/cadernobiologicas/article/ view/4602/2503

3. Pereira de Paiva MH, Pinheiro Lages L, Cavalcanti de Medeiros Z. Studies on forensic nursing in Brazil: a systematic review of the literature. In Nurs Rev. 2017 jun;64(2):286-95. http://dx.doi.org/10.1111/inr.12328. PMid:27682253.

4. Cachoeira DBC, Evangelista HRF, Souza WL. Enfermagem forense: contexto histórico, atuação do enfermeiro, contribuições para saúde e segurança pública [Internet]. Maceió: Centro Universitário Tiradentes - UNIT/AL; 2018 [citado 2021 mar 17]. Disponível em: http://openrit. grupotiradentes.com:8080/xmlui/handle/set/3223

5. Marcelo KCFRM, Barreto CA. Enfermagem forense sobre a regulamentação no Brasil. Revista Saúde em Foco [Internet]. 2019; [citado 2021 mar 17];11:1109-19. Disponível em: https://portal.unisepe.com.br/unifia/wpcontent/uploads/sites/10001/2019/05/050_ENFERMAGEM-FORENSE. pdf

6. Portaria n.. 204 , de 17 de fevereiro de 2016 (BR). Define a Lista Nacional de Notificação Compulsória de doenças, agravos e eventos de saúde pública nos serviços de saúde públicos e privados em todo o território nacional, nos termos do anexo, e dá outras providências. Diário Oficial da União, Brasília (DF), 2016: Seção 1.

7. Resolução n. 564, de 2017 (BR). Aprova o novo Código de Ética dos Profissionais de Enfermagem. Diário Oficial da União, Brasília (DF), 2017.

8. Rabêlo Alves JC, Jesus da Paz MJ. A importância da enfermagem forense para enfermeiros que atuam nas unidades de emergência. Revista Eletrônica Acervo Saúde. 2019;30(30):e1133. http://dx.doi. org/10.25248/reas.e1133.2019.

9. Xavier AAP, Silva EG. Assistência de enfermagem no atendimento de mulheres em situação de violência na atenção básica. Reicen [Internet]. 2019; [citado 2021 mar 17];2(Esp 2):293-300. Disponível em: https:// revistasfacesa.senaaires.com.br/index.php/iniciacao-cientifica/article/ view/279/217

10. Hazard D. The relevant physical trace in criminal investigation. Journa of Forensic Science and Medicine. 2016;2(4):208-12. http://dx.doi. org/10.4103/2349-5014.164662.

11. Rocha HN, Rodrigues BA, Paula GVN, Araújo JPA, Gomes TA, Souza ARN et al. $O$ enfermeiro e a equipe multidisciplinar na preservação de vestígios forenses no serviço de urgência e emergência. Brazilian Journal of Health Review.2020;3(2):2208-17. http://dx.doi.org/10.34119/bjhrv3n2-073.

12. Mendes KDS, Silveira RCCP, Galvão CM. Revisão integrativa: método de pesquisa para a incorporação de evidências na saúde e na enfermagem. Texto Contexto Enferm. 2008;17(4):758-64. http://dx.doi.org/10.1590/ S0104-07072008000400018.

13. Aromataris E, Munn Z. JBI manual for evidence synthesis. JBI, 2020 http://dx.doi.org/10.46658/JBIMES-20-01.

14. Melnyk BM, Fineout-Overholt E. Evidence-based practice in nursing \& healthcare. A guide to best practice. China: Wolters Kluwer/Lippincott Williams \& Wilkins; 2011.

15. Souza ACD, Martins IS, Silva JOM. O enfermeiro e a preservação de vestígios frente a violência sexual contra mulher. Revista Enfermagem da UNIT [Internet]. 2017; [citado 2021 Mar 17];1(1):1-4. Disponível em: https://eventos.set.edu.br/cie/article/viewFile/5366/1981

16. Silva JOM, Santos LFS, Dos Santos SM, da Silva DP, Santos VS, de Melo CM. Preservation of forensic evidence by nurses in a prehospital emergency care service in Brazil. J Trauma Nurs. 2020;27(1):58-62. http://dx.doi.org/10.1097/JTN.0000000000000483. PMid:31895321.

17. Campbell R, Greeson M, Patterson D. Defining the boundaries: how sexual assault nurse examiners (SANEs) balance patient care and law enforcement collaboration. J Forensic Nurs. 2011 mar;7(1):17-26 http://dx.doi.org/10.1111/j.1939-3938.2010.01091.x. PMid:21348930.

18. Dash SK, Patel S, Chavali K. Forensic nursing - Global scenario and Indian perspective. J Forensic Leg Med. 2016 ago;42:88-91. http:// dx.doi.org/10.1016/j.jlm.2016.05.020. PMid:27314972.

19. Heffron LC, Busch-Armendariz NB, Vohra SS, Johnson RJ, Camp V. Original research: giving sexual assault survivors time to decide: an 
exploration of the use and effects of the nonreport option. Am J Nurs. 2014 mar;114(3):26-35, quiz 36, 49. http://dx.doi.org/10.1097/01. NAJ.0000444489.49091.10. PMid:24518047.

20. Payne A. Sexual assault nurse examiner forensic examinations for immigrant victims: a case study. J Forensic Nurs. 2018;14(2):112-6. http://dx.doi.org/10.1097/JFN.0000000000000192. PMid:29781971.

21. Cowley R, WalshE, Horrocks J.The role of the sexual assault nurse examiner in england: Nurse experiences and perspectives.J Forensic Nurs. 2014;10(2):7783. http://dx.doi.org/10.1097/JFN.0000000000000026. PMid:24847871.

22. Du Mont J, Macdonald S, White M, Turner L, White D, Kaplan S et al. Client satisfaction with nursing-led sexual assault and domestic violence services in Ontario. J Forensic Nurs. 2014 jul-set;10(3):122-34. http:// dx.doi.org/10.1097/JFN.0000000000000035. PMid:25144583.

23. Downing NR, Mackin ML. The perception of role conflict in sexual assault nursing and its effects on care delivery. J Forensic Nurs. 2012;8(2):53-60. http://dx.doi.org/10.1111/j.1939-3938.2012.01135.x. PMid:22621663.

24. Delgadillo DC. When there is no sexual assault nurse examiner: emergency nursing care for female adult sexual assault patients. J Emerg Nurs. 2017;43(4):308-15. http://dx.doi.org/10.1016/j.jen.2016.11.006. PMid:28366240.
25. Bimber T. The Medical/Legal Aspects of Sexual Assault Nurse Examiner (SANE) Programs in Emergency Departments. Journal of Legal Nurse Consulting. 2014;25(1):32-5.

26. Cataruozolo PE. Forensic nursing: focus on SANE. Journal of Legal Nurse Consulting. 2015;26(1):33-7.

27. Santos FP, Eloi GVMA, Santos R, Santos BN, Silva JOM. Conhecimento e atuação de profissionais de saúde na preservação de vestígios forenses em pacientes vítimas de violência sexual em hospital de trauma em Aracaju. Anais do 2.. Congresso Internacional de Enfermagem. 13. Jornada de Enfermagem da Unit (JEU);2019 maio 6-10; Aracaju (SE), Brasil. Aracaju (SE): Unit; 2019.

28. Gomes AM. Preservação de vestígios no pré-hospitalar: papel das equipes de emergência médica em cenários forenses [Internet] Nursing; 2018 [citado 2021 Mar 17]. Disponível em: https://www.nursing pt/preservacao-de-vestigios-no-pre-hospitalar-papel-das-equipas-deemergencia-medica-em-cenarios-forenses/

29. de Oliveira Musse J, Santos VS, da Silva Santos D, Dos Santos FP, de Melo CM. Preservation of forensic traces by health professionals in a hospital in Northeast Brazil. Forensic Sci Int. 2020 jan;306:110057. http://dx.doi.org/10.1016/j.forsciint.2019.110057. PMid:31786515. 